\title{
Comparative Prevalence Studies of Hepatitis C Patients in Developing Vs Developed Countries
}

\author{
Umer Tahir1, 2, *Qurat-ul-Ain"1,2, Rai Waqas ${ }^{2,3}$, Irfan Bashir ${ }^{2,4}$ \\ ${ }^{1}$ Faculty of Pharmacy, The University of Lahore, Pakistan \\ ${ }^{2}$ Foundation for Young Researchers, Pakistan \\ ${ }^{3}$ Lahore College of Pharmaceutical Sciences, Lahore, Pakistan \\ ${ }^{4}$ Faculty of Pharmacy, University of Central Punjab, Lahore, Pakistan
}

\begin{abstract}
Viral hepatitis is one of the common transmissible causes of chronic liver responsible for increased risk of mortality and morbidity. Hepatitis $C$ disease has worldwide high prevalence which is more fatal because of lack of vaccination availability. Present study was conducted with the aim of to address the comparative prevalence and risk factor of hepatitis C. Secondary objective was to evaluate the prevalence of this disease in Pakistan as compared to other countries. Most of the countries in the world do not have significant studies of population infected with Hepatitis C virus (HCV). During 1990-2016, 232 studies were included; containing 117 countries representing that $90 \%$ of the world population have $2.3 \%$ prevalence. According to studies, developed countries have low HCV prevalence while developing countries have high prevalence. Pakistan being a developing country has second highest HCV prevalence ranging from 4.5 to $8 \%$. The reason of this high prevalence is unawareness about Hepatitis C in community and lack of successful therapies for patients with HCV. In past, developed countries had high prevalence but now they have low incidence because of proper system and awareness. Pakistan needs such kind of public awareness programs and better treatment facilities at affordable price to community.
\end{abstract}

Key Words: Viral, Transmissible, Fatal, Mortality, Morbidity, Vaccination, Community.

\section{INTRODUCTION}

Hepatitis is an inflammatory disease of liver having A, B, $\mathrm{C}, \mathrm{D}$, and $\mathrm{E}$ types. These types are very crucial because of the burden of illness and death they cause which depends upon potential for onset and epidemic uprising (WHO, 2016). Hepatitis $C$ virus chronically infected more than 170 million persons worldwide, and annually more than 350,000 persons die due to liver disease (Lawitz et al., 2013).

\section{HEPATITIS $C$}

It is a common chronic blood borne infection (Wilkins et al., 2010). Hepatitis C primarily infects hepatocytes, causing inflammation that later result in liver damage (Jacobson et al., 2010). Liver transplantation is majorly caused by it (Rich and Taylor, 2010). WHO estimates that minimum of $3 \%$ of the world's population is chronically infected with Hepatitis $\mathrm{C}$ virus (HCV). It causes infection in two phases, first involves acute attack that lasts for few weeks and if untreated then it may persist for longer time that is termed as chronic hepatitis C. The HCV belongs to the most successful of all persistent human viruses (Nawaz et al., 2015). $\mathrm{HCV}$ can represent as chronic or acute hepatitis. Asymptomatic hepatitis is acute form and advanced to hepatic failure rarely. Symptomatic hepatitis has a jaundice like clinical findings with $<25 \%$ of patient (Modi and Liang, 2008). About $85 \%$ of infected persons are raised to chronic hepatitis. There are 6 genotypes of HCV. Genetic ability and diversity of the virus to replicate rapidly may allow the hepatitis $C$ virus to evade the immune system of host (Cheryl Brower, 2012).

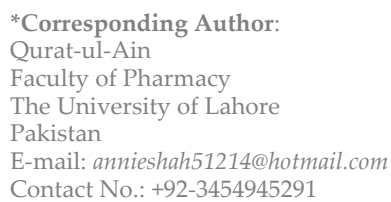

\section{TRASMISSION OF HCV}

Blood transfusion, use of parenteral, and unsafe sex (Meena et al., 2011) occupational, household, direct percutaneous inoculation and vertical transmission of HCV are the main modes of transmission (Elsheikh and Elsheikh, 2016). Among injection drug users sharing of contaminated needles, syringes, other medical equipment which are incompletely sterilized can also cause Hepatitis C (Wilkins, Malcolm et al. 2010). Heavy alcohol intake, particularly in females, may be associated with more rapid progression of different HCV liver diseases (Lavanchy, 2011).

\section{SYMPTOMS AND CLINICAL FINDINGS}

Majority of persons with chronic hepatitis $C$ will remain asymptomatic for decades and some for their entire lives, because of the slow progression of liver disease (Cheryl Brower, 2012). 50-90\% cases of Acute HCV infection are asymptomatic. According to the route of transmission failure to spontaneously eradicate infection occurs in 50-90\% of cases (Liver, 2011). During the acute phase of infection, clearance of HCV is typically associated with appearance of a vigorous T cell response, whereas evolution to chronicity is associated with poor response of T-cell (Loomba et al., 2011). Symptoms are nonspecific and include nausea, anorexia, weight loss, arthralgia, fatigue, myalgia, and weakness. In about 10 to 20 percent of patient chronic infection advanced to cirrhosis, and other complications including hepatocellualer carcinoma, ascites, hemorrhage and portal hypertension. HCV has deteriorating effect on whole body systems which produce exacerbating symptoms fever, epigastric pain, burning micturition, heart burn, burning palm and sole, chills, and fluid retention and muscle pain. These may also include generalized itching. There is also involvement of systemic (gastrointestinal), cognitive, mood and nervous systems in this disease to display the 
relative intensity of symptoms. It may also include hypoglycemia, hyperglycemia, chest pain, menstrual changes, palpitations and sexual anomalies (Nawaz et al., 2015) Portal hypertension and esophageal varices, spontaneous bacterial peritonitis, neurological complications such as hepatic coma, hepatic encephalopathy and hepatorenal syndrome leading to renal failure may occur in result of decompensated cirrhosis. It may also include testicular hypertrophy, non-Hodgkin lymphoma, Sjogren's syndrome, Raynaud's syndrome, palmar erythema, parotid enlargement and temporal muscle wasting (Modi and Liang, 2008). Recombinant immunoblot assay, quantitative HCV RNA polymerase chain reaction (PCR), and HCV antibody enzyme immunoassay are diagnostic tests used for the detection of HCV. Recombinant immunoblot assay detects antibodies to individual HCV antigens and has a higher specificity (Wilkins, Malcolm et al. 2010). In nearly $80 \%$ of cases within 15 weeks of exposure A serological blood test, Gold standard used to investigate the HCV (Nawaz et al., 2015). For the treatment of chronic HCV infection direct acting nucleotide polymerase inhibitor oral drug and interferons are used for 24 to 48 weeks to provide the standard care for all patients with HCV (Gane et al., 2013).

Recommendations for Control and Prevention of $\mathrm{HCV}$ Infection

Routine testing for $\mathrm{HCV}$ is recommended for:

- Persons with a history of injecting illegal drugs

- Persons on chronic hemodialysis

- Persons having persistent abnormal alanine aminotransferase level.

- Recipients of organ transplants and transfusion, including persons who received blood from $\mathrm{HCV}$ positive blood donor.

- Persons with known exposures, including emergency medical, Health care, and public safety workers after mucosal exposures to HCV-positive blood or needle sticks (Smith et al., 2012)

Key Recommendations for practice

- HCV infected persons should be vaccinated for Hepatitis $B$ and $A$.

- Alcohol intake should be avoided in persons infected with chronic HCV.

- Persons with chronic HCV infection should not use Hepatotoxic drugs.

- In Persons with chronic HCV infection and cirrhosis surveillance for hepatocellular carcinoma should be considered (Wilkins et al., 2010).

\section{PREVENTIVE MEASURES}

Preventing HIV transmission is one of the key challenges to health systems.

Following are the interventions for the prevention, treatment and care of HIV is:

- Safe Needle and syringe programs.

- Usage of Opioid substitution therapy and other drug dependence treatment.

- $\quad$ Effective HIV testing and counseling.

- Usage of appropriate antiretroviral therapy.

- Prevention and treatment of sexually transmitted infections.

- By conducting Condom programs for HCV sexual partners.

- Provide targeted information, education and communication and HCV sexual partners.
- Proper Vaccination, diagnosis and treatment of hepatitis C (WHO, 2012)

- Provision of sterile drug preparation equipment, Needle/syringe vending machines, bleach disinfection, the promotion of non-injecting routes of administration to prevent HCV (Cohen-Kashi Malina et al., 2009).

\section{PREVENTIVE}

Hepatitis $C$ virus is a basic pathogen and a major cause of morbidity and death. Whereas the cause rate of $\mathrm{HCV}$ infection is probably reducing in the developed world as compare to developing one, but deaths from liver disease supporting HCV infection will continue to increase in coming years (Messina et al., 2015). Chronic HCV infection is a cause of high prevalence of psychiatric co-morbidity in infected persons and latest information suggests that mental health problems may be associated with the infection (Schaefer et al., 2012).

\section{Total global HCV infections}

Total global prevalence of HCV was estimated to be $1.6 \%$ (1.3-2.1\%), according to 115 million past infected patients, 104 million were among adults (older than 15 years old) with infection rate of $2.0 \%$ (1.7- $2.3 \%)$ of HCV. 80 million viraemic infected patients showed prevalence to be $1.1 \%$ (0.9-1.4\%). Again, most of 75 million of adults gave the viraemic prevalence of 1.4\% (1.2-1.7\%) (Gower et al., 2014).

\section{GLOBAL PREVALENCE OF HCV}

Most serious and major disease burden in the world is the Hepatitis C. In year 1997, WHO surveyed virus affecting 170 million people worldwide with a global prevalence of about $3 \%$ and expected increase of 3 to 4 million new infected patients each year (Tiwari et al., 2010). Over the last 15 years, Hepatitis $C$ disease burden increased prevalence to $2.8 \%$, equating to $>185$ million infections globally. From 117 countries representing $90 \%$ of the world's population 1,217 studies were included in the analysis. The North America and Western Europe had the greatest number of studies. North Korea, Malaysia and Bangladesh are three most populous countries with no HCV information and data for the analysis.

To evaluate age-specific prevalence curves in 1990 and 2005, 232 articles were pooled to produce age-standardized prevalence globally and number of people with HCV has increased from 2.3\% (Mohd Hanafiah et al., 2013). The coverage and quality of population-based on HCV prevalence depends on an accurate diagnostic test and a representative population sample. To better evaluate the burden of chronic disease and the incidence trends; Gender, Age and Ethnicity are factors for the prevalence of HCV infection that should be stratified in proper manner (Lavanchy, 2011).

\section{Prevalence of HCV in Developed Countries}

A developed country is a sovereign state that has a highly developed economy and advanced technological infrastructure relative to other less industrialized nations. The incidence rate of $\mathrm{HCV}$ infection is apparently decreasing in the developed world, deaths from liver disease secondary to $\mathrm{HCV}$ infection will continue to increase over the next 20 years (Messina et al., 2015).

$\mathrm{HCV}$ prevalence in the developed countries is as follow: a) HCV in United State of America

United State is one of the largest continents of world with many counties. According to various studies, in 2001 prevalence of HCV peaked at 3.6 million (Davis et al., 2010). 
Another study show 3.49 million and 3.57 million infected persons in 1994 and 2001 respectively (Davis et al., 2010) showing a mild increase in United States. Further studies show prevalence of $1.6 \%$ or 4.1 million persons were HCV positive in US (Chak et al., 2011) showing a rapid increase in the infected patients. US has the hepatitis $C$ prevalence of $1.8 \%$ (Lavanchy, 2011). America a famous country, with total prevalence of $1.3 \%$ (95\% UI: $0.9 \%-1.9 \%$ ), which is likely similar but slightly higher than the 2005 results of $1.1 \%$ for the U.S (Mohd Hanafiah et al., 2013) All the studies and information show that in US there is slight negotiable increase in the HCV patient reported every year due to the increase rate of prevalence.

\section{b) HCV in France Community}

France is another most developed country with the Hepatitis $C$ virus prevalence of $2.4 \%$ (Mohd Hanafiah et al., 2013) and according to another survey conducted after a year in France HCV prevalence was about $0.6 \%$ (Gower et al., 2014) it show the decrease in the prevalence in the HCV patients. It is due to the good health facilities and controlled measures provided to cure the HCV.

\section{c) HCV in Malaysia Population}

Malaysia is also one developed country but its misfortune that no proper and regular studies are reported. One study was held in 2011, according to this Malaysia has HCV prevalence of 1.5\% (Lavanchy, 2011) which show slight amount of $\mathrm{HCV}$ patients.

\section{d) HCV in Japanese Community}

Japan has one of the highest endemic rates of HCV infection; approximately 2 million people are infected (McEwan et al., 2014). According to the studies of japan HCV prevalence is $1.4 \%$ (Mohd Hanafiah et al., 2013) and HCV prevalence is $1.5 \%$ (Gower et al., 2014) showing a constant value of the $\mathrm{HCV}$ patient with a slight variation due to proper and well equipped measures by Japan.

\section{e) HCV in Australian Community}

Australia is known to have lower HCV prevalence. In 2005 population of 264,000 with prevalence of $1.3 \%$ and 9700 are new infections in the same year. This incidence represented a drop from a peak of 14,000 in 1999 as the result of a reduction in IDU. Anti-HCV testing in 2800 samples showed an age-standardized prevalence of $2.3 \%$, (Sievert et al., 2011) HCV prevalence is $2.7 \%$ (Mohd Hanafiah et al., 2013) In Australia 1.7\% HCV prevalence was reported (Gower et al., 2014) Australia shows a good improvement and controlled role in the HCV prevalence and lower the HCV risk in its population from 2.7 to 1.7 .

\section{f) HCV in Canada Population}

In Canada HCV prevalence is 1.3\% (Mohd Hanafiah et al., 2013) which in 2014 reduce to $1.1 \%$ (Gower et al., 2014). These results show that Canada is progressing and working in the field of health to reduce the Hepatitis incidence to eradicate the disease.

\section{g) HCV in China}

In China, according to studies HCV prevalence was 1-1.9\%. Since 1992, several studies reported prevalence within a range of $0.29-9.6 \%$ (Sievert et al., 2011). Now in 2014, China has the reduced prevalence of $1.3 \%$ (Gower et al., 2014) concluding that China is the country with the most development and steps taken to reduce the infection rate of Hepatitis C.

\section{h) HCV Prevalence in Developing Countries}

Developing country also called as third world country, a less developed country or underdeveloped country, is a nation with a less developed industrial base, and a low $\mathrm{Hu}-$ man Development Index (HDI) relative to other countries.

$\mathrm{HCV}$ prevalence in the developing countries is as follow: a) $\mathrm{HCV}$ in Indian Community

India has Hepatitis C prevalence of $0.66 \%$, which decreased with time from $1.01 \%$ in 2000 to $0.29 \%$ in 2005 showing good change in prevalence (Sievert et al., 2011). India has the HCV prevalence of $1.5 \%$ (Lavanchy, 2011) resulting a huge increase in the Hepatitis $C$ infection and spread due to the poor and improper steps and precautions.

\section{b) HCV in Iran}

Iran has the HCV prevalence of $0.9 \%$ (Lavanchy, 2011) and in 2014 Iran show prevalence of $0.5 \%$ (Gower et al., 2014) being developing country Iran is taking proper precautions and preventing measures and it show the clear improvement in the country.

\section{c) HCV in Iraq}

Past studies show that Iraq has the HCV prevalence of 3.21\% (Lavanchy, 2011) and now in Iraq the HCV prevalence raised to $3.2 \%$ (Gower et al., 2014) valuating a huge increase in the Hepatitis C infection and spread due to the poor and improper measures and precautions.

\section{d) HCV in Afghanistan}

Afghanistan is to behind in the developing countries. Due to the tribal and the land area problems there are no proper working and studies based on the HCV prevalence. In past 1 study was held, Afghanistan showing HCV prevalence is $3.4 \%$ (Mohd Hanafiah et al., 2013) it shows a huge number of infected persons by the Hepatitis $C$ virus due to no precaution and preventive measures.

\section{e) HCV in Palestine}

Palestine is a poor developing country. In Palestine HCV prevalence is 3.6\% (Mohd Hanafiah et al., 2013) having the prevalence more than Afghanistan with more infected persons with worst medical facilities and preventive measures.

\section{f) HCV in Nepal}

Nepal is a South Asian country, with considerable diversity of people in ethnic group, socioeconomic condition and race (Tiwari et al., 2010) Nepal has the HCV prevalence of 0.64\% (Lavanchy, 2011).

\section{g) HCV in African population}

Africa has the highest reported HCV prevalence (Mohd Hanafiah et al., 2013) HCV prevalence is $2.8 \%$. Prevalence increases with increasing age until peak prevalence of $5.3 \%-6.7 \%$ reached at $55-64$ years in 2005 . Total prevalence decreased from $4.0 \%$ (95\% UI: 3.4-4.5\%) in 1990 to $2.8 \%$ (95\% UI: 2.4\%-3.3\%) in 2005 (Mohd Hanafiah et al., 2013) Now Africa has the decreased prevalence of $1.6 \%$ (Gower et al., 2014).

\section{h) HCV in Pakistan}

Pakistan the $6^{\text {th }}$ most populous country in the world with the population of 16,094,3000 (Umar and Bilal, 2012) Pakistan ranging from $4.5 \%$ to $8.0 \%$ has the second highest prevalence rate of hepatitis C (Jiwani, 2011) 132 published studies from 1992 to 2008 were pooled and prevalence of 
$4.7 \%$ in the general population and of $3 \%$ among blood donors. In 2008 published review reported that Pakistan has HCV prevalence between 2.4 and 6.5\% (Sievert et al., 2011). Prevalence of HCV infection reported in various studies by the Pakistani authors ranges from $2.2-14 \%$. To an estimate 10 million people in Pakistan are infected with Hepatitis C virus. Pakistan four provinces have varied HCV prevalence: as in Punjab, Baluchistan, Sindh and Khyber Pakhtunkhwa with $\mathrm{HCV}$ prevalence of $6.7 \%, 1.5 \%, 5 \%$ and $1.1 \%$, respectively. Prevalence of $4.7 \%$ in healthy adults (Umar and Bilal, 2012). In Punjab, estimated prevalence of Hepatitis $C$ is $6.5 \%$. While the Hepatitis B and C in general population National Survey on the prevalence of Pakistan (2008) measured HCV, prevalence is 4.9\% (Gorar and Zulfikar, 2010). In 2014 the prevalence of Pakistan raised to $6.7 \%$ (Gower et al., 2014). Pakistan represents variation in $\mathrm{HCV}$ prevalence as the population is quite heterogeneous due to factors such as disease duration, gender and age at infection; therefore, the course of disease is nonlinear and variable over time. Following are the factors for the high HCV prevalence as Economic Survey of Pakistan 2005 to 2006; the government spent 0.75 percent of GDP on health sector. With a low allocation of funds. The public health sector lacks quality services, few doctors are certified while most are unregistered. On per 1000 population, Pakistan has just 7.3 physicians and 4.7 nursing personnel in the health care setup. These professionals also lack motivations to work regarding their salary, allowance and fund package (Jiwani, 2011).

\section{CONCLUSION}

$\mathrm{HCV}$ is one of the most commonly transmissible fatal diseases with a high global prevalence. In developed countries, its prevalence was high but now there is low incidence of $\mathrm{HCV}$ because they have proper public health awareness programs and better treatment facilities are available for HCV. Pakistan being a developing country has high prevalence and it is increasing day by day because there are no proper preventive measures available and lack of public awareness programs and treatment facilities for HCV patients. The other reason of high prevalence is nonaffordable prices of HCV treatment to community.

\section{Recommendations for Ministry of Health}

- The Ministry of Health should play important role on bringing HCV on the priority agenda and to obtain political commitment within the government and other relevant ministries to lower the HCV prevalence in $\mathrm{Pa}$ kistan.

- Efficient research activity on different harm reduction interventions to contribute to the emerging evidence base as there is limited evidence.

- To measure the effectiveness of prevention measures, develop an effective surveillance system to monitor trends in HCV infection.

- Emphasize infection control and blood safety mechanisms.

- Intentions and Efforts of raising awareness on HCV in IDUs as well as in general population are needed to be raised up.

- By Forming good models to estimate the impact of harm reduction interventions on transmission of $\mathrm{HCV}$.

- Decrease the use of both direct and indirect sharing of syringes or other injecting equipment.

- Behavioral and psychological Counseling of infected persons as an attempt to prevent transmission of $\mathrm{HCV}$ infection to other healthy persons.
- Improving the accessibility and effectiveness of harm reduction services to reduce $\mathrm{HCV}$ prevalence.

- To respond the comprehensive service needs of IDUs in both public and private health sectors a Legislation should be done.

\section{Recommendations for Donors}

- $\quad$ Financing gaps must be filled for HCV prevention and other health care services and systems.

- Support health systems evaluation and monitoring. It is still needed to identify the most effective primary prevention intervention to reduce transmission of $\mathrm{HCV}$ infection in persons, especially in injecting drug users.

\section{Recommendations for NGOs}

- Mechanisms in governance, increased capacity and transparency accountability is crucial.

- Extent of implementation of coordination and programs need to be upgrade.

- Intensive assistance or advocacy with political leadership is required to make them realize the $\mathrm{HCV}$ as public health problem has high prevalence and for support in response of it.

- $\quad$ Facilitating comprehensive policy dialogue involving NGO sector, public society, politicians, and public departments.

\section{REFERENCES}

Chak, E., Talal, A.H., Sherman, K.E., Schiff, E.R., Saab, S., (2011). Hepatitis $c$ virus infection in USA: An estimate of true prevalence. Liver international, 31(8), 1090-1101. [DOI]

Cheryl Brower, R., Msph, (2012). Hepatitis and hemophilia. 1-20.

Cohen-Kashi Malina, K., Cooper, I., Teichberg, V.I., (2009). Closing the gap between the in-vivo and in-vitro blood-brain barrier tightness. Brain Res, 1284, 12-21. [DOI]

Davis, G.L., Alter, M.J., El-Serag, H., Poynard, T., Jennings, L.W., (2010). Aging of hepatitis c virus (hcv)-infected persons in the united states: A multiple cohort model of hcv prevalence and disease progression. Gastroenterology, 138(2), 513-521. e516. [DOI]

Elsheikh, M.A., Elsheikh, R.M., (2016). O268 hepatitis b virus and hepatitis $\mathrm{C}$ virus in pregnant sudanese women.

Gane, E.J., Stedman, C.A., Hyland, R.H., Ding, X., Svarovskaia, E., Symonds, W.T., Hindes, R.G., Berrey, M.M., (2013). Nucleotide polymerase inhibitor sofosbuvir plus ribavirin for hepatitis c. New England Journal of Medicine, 368(1), 34-44. [DOI]

Gorar, Z.A., Zulfikar, I., (2010). Seropositivity of hepatitis c in prison inmates of pakistan--a cross sectional study in prisons of sindh. J Pak Med Assoc, 60 (6), 476-479. PMid:20527648

Gower, E., Estes, C., Blach, S., Razavi-Shearer, K., Razavi, H., (2014). Global epidemiology and genotype distribution of the hepatitis $\mathrm{c}$ virus infection. Journal of hepatology, 61(1), S45-S57. [DOI]

Jacobson, I.M., Cacoub, P., Dal Maso, L., Harrison, S.A., Younossi, Z.M., (2010). Manifestations of chronic hepatitis $c$ virus infection beyond the liver. Clinical Gastroenterology and Hepatology, 8(12), 1017-1029. [DOI]

Jiwani, N., (2011). A silent storm: Hepatitis c in pakistan.

Lavanchy, D., (2011). Evolving epidemiology of hepatitis c virus. Clinical Microbiology and Infection, 17(2), 107-115. [DOI]

Lawitz, E., Mangia, A., Wyles, D., Rodriguez-Torres, M., Hassanein, T., Gordon, S.C., Schultz, M., Davis, M.N., Kayali, Z., Reddy, K.R., (2013) Sofosbuvir for previously untreated chronic hepatitis c infection. New England Journal of Medicine, 368(20), 1878-1887. [DOI]

Liver, E.a.F.T.S.O.T., (2011). Easl clinical practice guidelines: Management of hepatitis c virus infection. Journal of hepatology, 55(2), 245-264.

Loomba, R., Rivera, M., Mcburney, R., Park, Y., Haynes-Williams, V., Rehermann, B., Alter, H., Herrine, S., Liang, T., Hoofnagle, J., (2011). The natural history of acute hepatitis c: Clinical presentation, laboratory findings and treatment outcomes. Alimentary pharmacology \& therapeutics, 33(5), 559-565. [DOI] 
Mcewan, P., Ward, T., Webster, S., Yuan, Y., Kalsekar, A., Broglio, K., Kamae, I., Quintana, M., Berry, S.M., Kobayashi, M., (2014). Estimating the long-term clinical and economic outcomes of daclatasvir plus asunaprevir in difficult-to-treat japanese patients chronically infected with hepatitis c genotype $1 \mathrm{~b}$. Value in Health Regional Issues, 3, 136145. [DOI]

Meena, M., Jindal, T., Hazarika, A., (2011). Prevalence of hepatitis b virus and hepatitis c virus among blood donors at a tertiary care hospital in india: A five-year study. Transfusion, 51(1), 198-202. [DOI]

Messina, J.P., Humphreys, I., Flaxman, A., Brown, A., Cooke, G.S., Pybus, O.G., Barnes, E., (2015). Global distribution and prevalence of hepatitis c virus genotypes. Hepatology, 61(1), 77-87. [DOI]

Modi, A., Liang, T., (2008). Hepatitis c: A clinical review. Oral diseases, $14(1), 10-14$. [DOI]

Mohd Hanafiah, K., Groeger, J., Flaxman, A.D., Wiersma, S.T., (2013) Global epidemiology of hepatitis c virus infection: New estimates of age-specific antibody to hcv seroprevalence. Hepatology, 57(4), 1333 1342. [DOI]

Nawaz, A., Zaidi, S.F., Usmanghani, K., Ahmad, I., (2015). Concise review on the insight of hepatitis c. Journal of Taibah University Medical Sciences, 10(2), 132-139. [DOI]

Rich, J.D., Taylor, L.E., (2010). The beginning of a new era in understanding hepatitis c virus prevention. Journal of Infectious Diseases, 202(7), 981-983. [DOI]
Schaefer, M. Capuron, L, Friebe, A, Diez-Quevedo, C, Robaeys, G, Neri, S., Foster, G.R., Kautz, A., Forton, D., Pariante, C.M., (2012). Hepatitis c infection, antiviral treatment and mental health: A european expert consensus statement. Journal of hepatology, 57(6), 1379-1390. [DOI]

Sievert, W., Altraif, I., Razavi, H.A., Abdo, A., Ahmed, E.A., Alomair, A., Amarapurkar, D., Chen, C.H., Dou, X., El Khayat, H., (2011). A systematic review of hepatitis c virus epidemiology in asia, australia and Egypt. Liver International, 31(s2), 61-80. [DOI]

Smith, B.D., Morgan, R.L., Beckett, G.A., Falck-Ytter, Y., Holtzman, D., Teo C.-G., Jewett, A., Baack, B., Rein, D., Patel, N., (2012). Recommendations for the identification of chronic hepatitis $\mathrm{c}$ virus infection among persons born during 1945-1965. MMWR Recomm Rep, 61(RR-4), 1-32. PMid:22895429

Tiwari, B., Ghimire, P., Kandel, S., Rajkarnikar, M., (2010). Seroprevalence of hbv and hcv in blood donors: A study from regional blood transfusion services of Nepal. Asian journal of transfusion science, 4(2), 91 [DOI]

Umar, M., Bilal, M., (2012). Hepatitis c, a mega menace: A Pakistani perspective. Journal of Pioneering Medical Sciences, 2(2), 68 .

WHO (2012).

WHO (2016)

Wilkins, T., Malcolm, J.K., Raina, D., Schade, R.R., (2010). Hepatitis c: Diagnosis and treatment. Am Fam Physician, 81(11), 1351-1357. PMid:20521755 